\title{
THE PREVALENCE OF EUSTACHIAN VALVE ON TRANSOESOPHAGEAL ECHO EXAMINATION
}

\author{
Dan Marek*, Eliska Sovova, Eva Kocianova
}

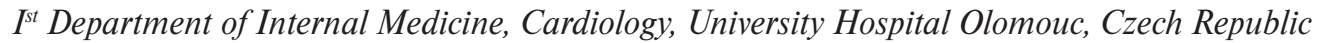 \\ E-mail:audiodan@centrum.cz
}

Received: January 18, 2011; Accepted: March 22, 2011

Key words: Eustachian valve/Prevalence/Transoesophageal echocardiography/Complications

Background. The Eustachian valve (EV) is an embryonic structure redirecting the blood flow from the inferior vena cava through the foramen ovale. It may persist in adults as a floating membrane in the right atrium. A large EV can be associated with thrombosis, embolization, bacterial endocarditis, etc. The prevalence of EV in the normal population is unknown.

Aim. to assess the prevalence and size of EV in unselected patients examined by transoesophageal echo.

Methods. 1100 consecutive patients were examined for various reasons by a single operator and interpreter. Routine check was made for the presence of EV in the right atrium. Only patients with a mobile EV and not with a simple rigid rim were counted. Three measurements of the EV length at the level of the aortic valve (i.e. $90^{\circ}$ ) were made off-line and the longest distance considered. If the EV was not visualized and recorded as a straight line because of its chaotic movement, the picture of the valve was segmented into several separated valve portions and the sum taken as the true length.

Results. 46 patients (24 males) with EV were identified out of total 1100 pts examined. The average length of the EV was $23.6 \pm 9.7 \mathrm{~mm}(7-47 \mathrm{~mm}$, median $23 \mathrm{~mm})$.

Conclusions. The finding of a persisting Eustachian valve on transoesophageal echo examination is not rare. The prevalence was similar in men and in women. Bearing in mind the possible complications associated with a large valve, we recommend that a proper description of persistent EV is included in routine transoesophageal echo reports.

\section{INTRODUCTION}

The Eustachian valve (EV) is a structure that can be noted in the right atrium in some individuals. It was described by Eustachius in 1563. In a normal embryo it is a membrane which extends from the inferior vena cava to the lower part of the fossa ovalis, redirecting the blood flow from the inferior vena cava through the atrial septum to the left atrium. Just a small remnant of the EV usually persists in adults and is considered a normal variant. In some people however, this structure is prominent and can be visualized on echo (even transthoracic) as a mobile membrane in the right atrium (Fig. 1). The length of the EV can be up to several centimeters (depending on the echo projection). If a prominent EV persists, complications may occur such as obstruction of the inferior vena cava, thrombosis and possibly subsequent pulmonary embolism, infective endocarditis, etc. The prevalence of EV in the general population is not known very well, as the diagnostic criteria vary in different studies.

\section{AIM}

To determine the prevalence and size of $\mathrm{EV}$ in patients examined by transoesophageal echocardiography.

\section{METHODS}

The sample included 1100 consecutive patients who were being examined for a variety of reasons by transoesophageal echo (TEE) by a single operator and interpreter in a single echocardiography laboratory at a tertiary cardiac centre. The general indications for TEE examination included "shunt identification", "cardiac source of embolism", "bacterial endocarditis", or "poor transthoracic image quality". A Toshiba Power Vision 8000 ultrasound machine with $7.5 \mathrm{MHz}$ multiplane transoesophageal probe was used for the investigation. The patients were examined under topical anesthesia \pm mild sedation (midazolam i.v.). Routine check was made for the presence of EV in the right atrium during each examination. Multiple loops at the level of the aortic valve (i.e. $90^{\circ}$ ) were recorded. The length of the EV was assessed off-line on the frozen picture. Three measurements were taken for each patient and the longest distance was counted. If the EV was not visualized and recorded as a straight line because of its chaotic movement, the picture of the valve was segmented into several separated valve portions, the sum being taken as the true length (Fig. 2).

\section{RESULTS}

There were no complications during the TEE procedures. Of 1100 consecutive patients, right atrial mobile 

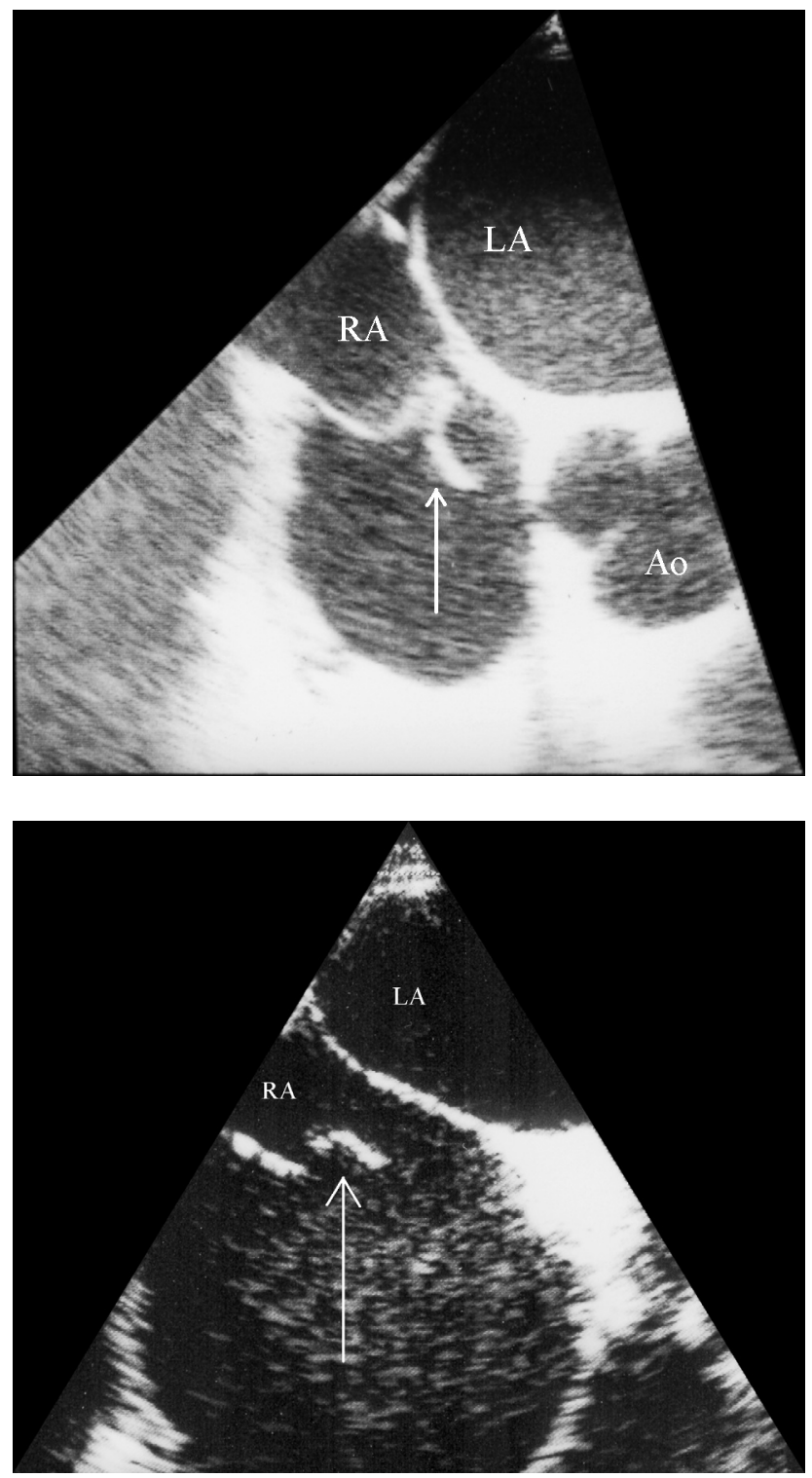

Fig. 1. Two examples of a big Eustachian valve floating in the right atrium (arrow), as seen on transoesophageal echo examination.

Ao: aorta; LA: left atrium; RA: right atrium

structure meeting the criteria of the Eustachian valve was identified in forty six patients (4.2\%). This subset consisted of 24 males and 22 females (average age $55 \pm 16$, range 26-83). There were no patients in whom the findings were classified as Chiari network. The average length of the EV was $23.6 \pm 9.7 \mathrm{~mm}(7-47 \mathrm{~mm}$, median $23 \mathrm{~mm})$.

\section{DISCUSSION}

The EV is a physiological structure in newborn children which usually disappears during the first years of life. Its appearance in adults varies between "nothing or almost nothing" to "a very long, large and potentially clinically significant structure", with no strict criteria for its "presence" or "absence". A simple Eustachian valve could be differentiated from a complex Chiari network which was reported by Chiari in 1897 - the network appeared as a perforated membrane of various shapes. While the Eustachian valve was usually derived only from the right venous valve, Chiari postulated that networks were derived also from the embryonic septum spurium ${ }^{1}$, the most prominent anterior pectinate muscle of the crista terminalis. An extensive investigation of 100 necropsies made by Powell and Mullaney showed that in 24 cases, the venous valve was either fenestrated or replaced by fibres. Interestingly, in 12 cases there was a continuity of tissue between the Eustachian and Thebesian valves ${ }^{1}$, i.e. the valves covering the coronary sinus ostium. Yater published a review on valve variations, based on the autopsy of " 120 routinely collected hearts, all of which would be considered developmentally normal. A Eustachian valve, or valve of the inferior vena cava, was found to be present in some form in all but seventeen of these hearts" - $86 \%$ $\left(\right.$ ref $\left.^{2}\right)$. The echocardiographic features of Chiari network were described in $1981\left(\mathrm{ref}^{3}\right)$.

Identification of the Eustachian valve is not a major issue in most echocardiographic studies. The EV is usually considered to be a normal variant, and as such, its presence is not mentioned in routine echo reports. For this reason, the prevalence of EV in adult echocardiography is not known. Limacher et al. ${ }^{4}$ published an echocardiographic study in pediatric patients. The EV was visualized in 46 of 54 children younger than 1 month and in 61 of 91 older children (mean age $3.3 \pm 2$ years).

The presence of a large EV is usually an innocent echo finding. In some situations, however, it may be clinically significant. Complications associated with a large EV include obstruction of the inferior vena cava ${ }^{5}$, thrombosis ${ }^{6,7}$ and possibly subsequent pulmonary embolism ${ }^{6}$. Infective endocarditis $^{8,9}$ is a major issue; the involvement of persistent $\mathrm{EV}$ in infective endocarditis is often described in drug abusers. Sapin described a rare case of a patient with an atrial septal defect, in whom the EV was mistaken for the lower rim of the defect. The EV was sutured to the upper rim of the defect and this caused postoperative shortness of breath and apparent systemic blood oxygen desaturation $^{10}$. The Eustachian valve can also create difficulties for patients indicated for patent foramen ovale closure by Amplatzer device ${ }^{11}$. It can complicate permanent pacemaker implantation procedures as well, when the introduced pacemaker lead is trapped into the prominent valve $^{12}$. If a patent foramen ovale coexists, the EV may also be a risk factor for paradoxical embolization. In the literature, small samples of patients with brain embolism and increased prevalence of EV in comparison with a control group have been described. In an Austrian study, the EV was more common in patients with presumed paradoxical embolism than in control patients: 143 of 211 (68\%) vs. 31 of 95 (33\%), respectively, $P<0.001\left(\right.$ ref $\left.^{13}\right)$. In this study, there were no "size" criteria for the "presence" of EV. This explains the much greater prevalence of EV than we found. The height of EV in Limacher's study ranged from 2 to $20 \mathrm{mms}$ in their series, but a sagittal subcostal view was used for the measurement ${ }^{4}$. This view is often not available in adults because of their body build. Moreover, we measured only the mobile part of EV in our 


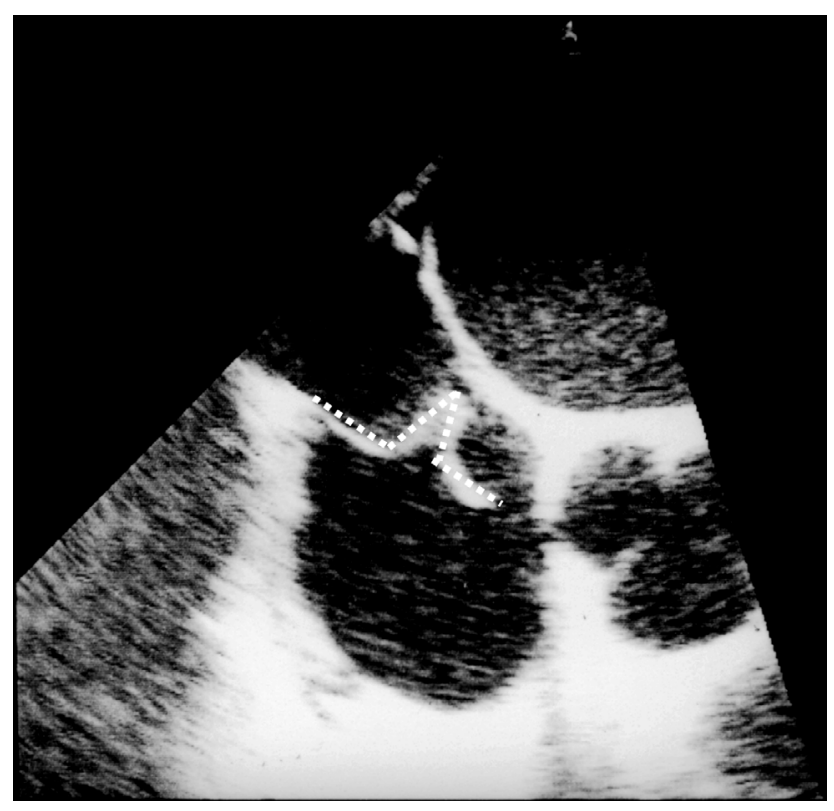

Fig. 2. Measurement of the length of a large Eustachian valve, where the valve was not visualized as a straight structure.

study. If patients with a fixed rim only had been included, a higher prevalence and smaller EV values would have been expected.

A major limitation of our study was that most patients examined were also patients with various cardiac diseases. A substantial number had stroke in their histories. This fact may have been reflected in the data. In the general population, the prevalence of a prominent EV may be slightly lower. However, TEE is a semi-invasive procedure and it would be difficult to design a large study on a "normal" healthy population to assess the true incidence of EV. The screening for EV as a primary objective would not justify such a study. The sample described in our study is not from a "normal" healthy population. Thus, we cannot generalize our findings of a prevalence of $4.2 \%$ of 1100 patients to any normal healthy population. One study that could be done to determine the clinical significance of an $\mathrm{EV}$ in relation to a stroke is evaluation of a larger patient sample where the incidence of stroke is compared for two groups of pts (EV+ versus EV-).

\section{CONCLUSION}

We demonstrated that the finding of a persisting Eustachian valve in transoesophageal echo examination was not very rare $(4.2 \%)$ and its prevalence was similar in men and in women. Bearing in mind all the possible complications associated with a large valve, we recommend that if the EV is found, its proper description should be included in routine transoesophageal echo report.

\section{REFERENCES}

1. Powell ED, Mullaney JM. The Chiari network and the valve of the inferior vena cava. Br Heart J 1960;22:579-84.

2. Yater, W. M. Variations and anomalies of the venous valves of the right atrium of the human heart. Arch Pathol 1929;7:418-441.

3. Werner JA, Cheitlin MD, Gross BW, Speck SM, Ivey TD. Echocardiographic appearance of the Chiari network: differentiation from right-heart pathology. Circulation 1981;63:1104-09.

4. Limacher MC, Gutgesell HP, Vick GW, Cohen MH, Huhta JH. Echocardiographic anatomy of the Eustachian valve. Am J Cardiol 1986;57:363-65.

5. Burton DA, Chin A, Weinberg PM, Pigott JD. Identification of cor triatriatum dexter by two-dimensional echocardiography. Am J Cardiol 1987;60:409-10.

6. Onwuanyi AE, Brown RJ, Vahedi M, Narayanan R, Nash IS, Goldman ME. Eustachian valve thrombus:critical factor in outcome of venous thromboembolism. Echocardiography 2003;20:7173 .

7. Barriales V, Tamargo JA, Aguado MG, Martin M, Rondan J, Segovia E, Morís C. Floating thrombi on the Eustachian valve as a complication of venous thromboembolic disease. Int J Cardiol 2004;93:289-91.

8. Wong RC, Teo SG, Yeo TC. An unusual right-sided endocarditis: a case report of eustachian valve endocarditis. Int J Cardiol 2006;109:406-07.

9. Sawhney N, Palakodeti V, Raisinghani A, Rickman LS, DeMaria AN, Blanchard DG. Eustachian valve endocarditis: a case series and analysis of the literature. J Am Soc Echocardiogr 2001;14:113942.

10. Sapin PM, Salley RK. Arterial desaturation and orthodeoxia after atrial septal defect repair: demonstration of the mechanism by transesophageal and contrast echocardiography. J Am Soc Echocardiogr 1997;10:588-92.

11. Butera G, Montinaro A, Carminati M. The "pull-push" technique to deal with a redundant eustachian valve interfering with placement of a PFO occluder. Catheter Cardiovasc Interv 2006;68:961-64.

12. Dissmann R, Schroder J, Voller H, Behrens S. Entrapment of pacemaker lead by a large net-like Eustachian valve within the right atrium. Clin Res Cardiol 2006;95:241-43.

13. Schuchlenz HW, Saurer G, Weihs W, Rehak P. Persisting eustachian valve in adults: relation to patent foramen ovale and cerebrovascular events. J Am Soc Echocardiogr 2004;17:231-33. 
